\title{
JOGOS ELETRÔNICOS E SUA EVOLUÇÃO
}

\author{
Anderson Cassio Franciscoํ․ Fernanda Maria de Souza², Alessandro Arraes Rodrigues ${ }^{3}$, \\ Hudson Sérgio de Souza ${ }^{4}$
}

\begin{abstract}
${ }^{1}$ Acadêmico do curso de Sistemas para Internet na Faculdade UniFatecie, e-mail: ander_sac 16@hotmail.com
${ }^{2}$ Acadêmica do curso de Sistemas para Internet na Faculdade UniFatecie, e-mail: ferrnanda-m@outlook.com

${ }^{3}$ Especialista em Educação. Coordenador e Docente do curso de Sistemas para Internet, da Faculdade UniFatecie Paranavaí, e-mail: alessandro.arraes@fatecie.edu.br

${ }^{4}$ Doutor em agronomia com ênfase em Agro-Informática, Bacharel em Sistemas de Informação, Docente do curso de Sistemas para Internet da Faculdade UniFatecie Paranavaí, email: hudson.souza@fatecie.edu.br
\end{abstract}

\section{RESUMO}

Os jogos eletrônicos passaram por uma grande evolução desde o seu primeiro game. As tecnologias e linguagens de programação utilizadas nos primeiros jogos são diferentes dos jogos atuais, podendo se considerar uma viagem no tempo. Atualmente existem jogos digitais para variadas plataformas, como por exemplo os vídeos games/consoles.

PALAVRAS-CHAVE: Games; Tecnologia; História.

\section{INTRODUÇÃO}

Esse artigo tem como objetivo apresentar a evolução dos jogos eletrônicos, desde o "Tennis for Two" até os consoles da atualidade. A indústria eletrônica ao passar dos anos vem crescendo com um faturamento de bilhões de dólares, e está sempre em constante melhoria, como o hardware avançado (nos consoles) e gráficos impecáveis em jogos digitais.

Desde a criação dos primeiros jogos eletrônicos, do joystick, e ATARI até consoles atuais como o Wii e o Xbox, os games ganham constantes aperfeiçoamentos. A evolução dos jogos eletrônicos está ligada intrinsecamente à evolução de aparatos e linguagens.

A narrativa e interatividade são fatores comparativos que torna essa categorização evidente. $\mathrm{O}$ artigo busca também bases para o entendimento histórico desta mídia. $\mathrm{A}$ tal congrega dentro do seu processo produtivo as habilidades dos campos intensificando o surgimento e a legitimação de uma nova mídia interativa que, advém da evolução tecnológica e da intensificação de teorias.

\section{JOGOS ELETRÔNICOS}

Com os jogos eletrônicos podemos entrar em um plano diferente, a possibilidade de ser um atirador de elite ou até mesmo aquele mago que derrota vários monstros em algumas dimensões. Toda essa possibilidade existe por conta dos computadores, consoles e mobiles, cada um com seu mercado e tecnologia para rodar seus jogos.

Os jogos utilizam toda tecnologia de um computador, sendo possível jogar em celulares, computadores, consoles e fliperamas. Existe uma evolução constante e com isso os games viraram uma forma de entretenimento bem popular, proporcionando diversão e outros benefícios (BRITANNICA, 2019).

\subsection{EVOLUÇÃO - DÉCADA DE 80}

A tecnologia mudou radicalmente no início dos anos 80 pela entrada da ibm no mercado de computadores pessoais (PEPE, 2017).

A IBM PCs ainda dominava o mercado, mas sofreram mudanças. Em 1985 a Intel lançou o processador i386, que foi adotado pela Compaq em seu DeskPro 386 - era um 
clone de PC melhor que as máquinas da IBM. Com isso, a ibm perdeu seu controle do mercado para empresas criando clones de PC (PEPE, 2017).

A segunda metade dos anos 80 veio muitas inovações tecnológicas que revitalizaram toda a indústria se preparando para uma explosão criativa nos anos 90 (PEPE, 2017).

\subsection{EVOLUÇÃO - DÉCADA DE 90}

A década de 90 foi a época que foram marcados por muita inovação. Jogos de vários gêneros foram criados que por sua vez marcou essa geração.

Dune Il definiu o padrão de jogos RTS, Wolfenstein 3D e Doom introduziram os FPS (ou "clones de Doom", como eram conhecidos), Civilization popularizou os jogos 4x, Alone in the Dark criou o survival horror, Street Fighter II, Mortal Kombat e The King of Fighters dominaram os arcades, Super Mario World, Donkey Kong Country, Sonic, Megaman X, Castlevania: Rondo of Blood e Super Metroid são lendas do estilo plataforma; de JRPGs tivemos Earthbound, Final Fantasy VI, Breath of Fire, Secret of Mana; de adventure tivemos duzias de clássicos da LucasArts e Sierra, Ultima Underworld mostrou ao mundo como fazer um jogo 3D; e a lista continua: Need for Speed, Warcraft, SimCity 2000, Mario Kart, X-COM, Lemmings, F-Zero, Wing Commander, Star Fox, etc. Com mais pessoas tendo acesso a computadores e modems, jogos "shareware" começaram a espalhar - epic pinball, duke nukem e doom, além de vários ferramente de software, podiam ser experimentados gratuitamente - depois comprados via correio (PEPE, 2017).

\subsection{EVOLUÇÃO - DÉCADA DE 2000}

Os anos 2000 foram marcados por as grandes empresas q controlavam como a EA, Activision e Ubisoft, além de três fabricantes de consoles - Nintendo, Sony e Microsoft. Assim os melhores jogos logo viravam "franquias", que passavam a ter lançamentos anuais, assassin`s creed e cal of duty são alguns deles (Quadro 1) (PEPE, 2017).

Quadro 1: Desenvolvedores com seus jogos e suas vendas

\begin{tabular}{|l|l|l|}
\hline DESENVOLVEDORES & JOGOS & VENDAS \\
\hline Ubisoft & Far Cry, Assasin's Creed e Just Dance & US $\$ 1,65$ bilhão \\
\hline Activition Blizzard & World of Warcraft, Diablo e Hearthstone & US $\$ 4,41$ bilhões \\
\hline Nitendo & Mario, Zelda, Pokémon e Smash Bros & US $\$ 4,61$ bilhões \\
\hline Capcom & Resident Evil e Street Fighter & US $\$ 500$ milhões \\
\hline
\end{tabular}

Fonte: Dados da pesquisa

O Gráfico 1 demostra o faturamento das empresa em bilhões de dólares. 


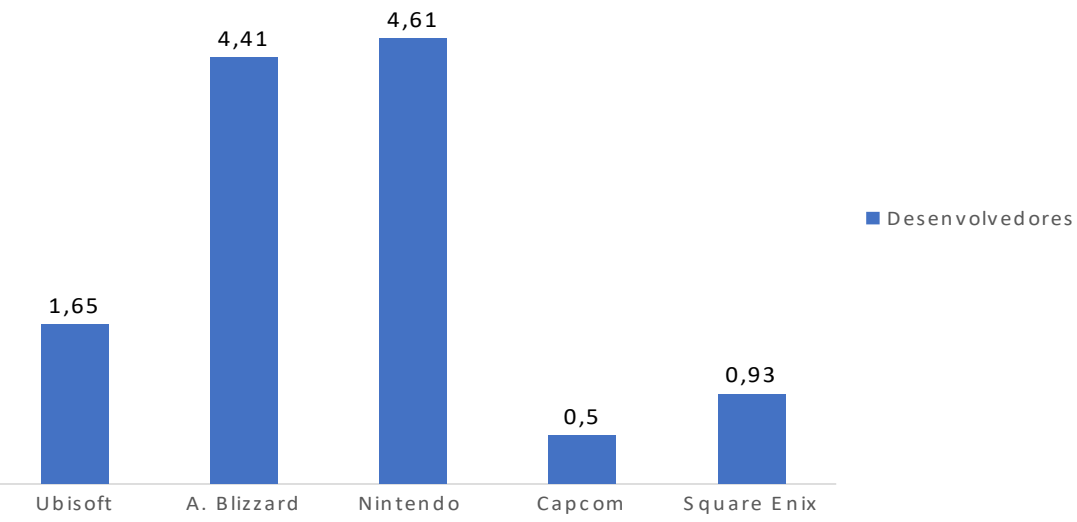

Gráfico 1: Venda em bilhões de dólares, por desenvolvedores de jogos digitais Fonte: Dados da pesquisa

Em 2010 a distribuição digital cresceu muito com o Steam dominando o mercado. O Greenlalight, introduzido em $2012 \mathrm{fez}$ com que qualquer desenvolvedor pudesse vender seus jogos, o Early Access introduzido em 2013 permitiu que vendessem jogos incompletos. Outro fator importante foi a popularização de jogos indie e mobile, que mudaram a percepção do público. Jogos hoje podem vir de um único desenvolvedor, de um estúdio médio ou de grandes equipes (PEPE, 2017).

\subsection{O QUE VEIO COM A EVOLUÇÃO DOS JOGOS}

Os games junto com sua evolução e popularização não trouxe só a diversão, mas também campeonatos. Por volta da década de 70 houve uma competição esportiva eletrônica na Universidade de Stanfor, o jogo se chamava Spacewar. Com essas competições se inicia a criação da eSport. Só a partir dos anos $90 \mathrm{com}$ progresso da internet que os games online para computadores ganham um marco e surge, o esportista eletrônico, os primeiros pro player (WOLNEI, 2018).

Esports, E-sports ou eSports é a abreviação para Eletronic Sports ou esportes eletrônicos, que na prática são competições profissionais de jogos de computadores ou videogames transmitidos para milhares de pessoas. O Esports veio para mostrar que já foi o tempo em que jogos de videogames e computadores são "coisas de criança". Hoje nós vemos um cenário que movimenta mais de 905 milhões de dólares (dados de 2018) em que estão envolvidos as empresas dos jogos, times, jogadores, transmissão e empresas que apoiam esse mercado para crescer (IGOR, 2019).

\subsection{MATERIAL DE MÉTODOS}

Este trabalho foi elaborado a partir de uma revisão de literatura com caráter descritivo, iniciando de modo seletivo sobre livros, revistas e artigos eletrônicos sobre o tema, utilizando-se das bases de dados do Google Acadêmico, Scielo, Periódicos Capes e Minha Biblioteca no período de Fevereiro a Julho de 2019. As buscas foram realizadas através das palavras-chave "Games", "tecnologia" e "história", assim como suas correspondentes em língua inglesa. Como critério de exclusão foram eliminados artigos anteriores ao ano de 2010. Agrupando todas as bases de dados, foram elencados 10 artigos com a finalidade do estudo. Seguido de uma análise crítica e analítica sobre os dados levantados, a fim de sintetizar e organizar as informações. 


\section{CONCLUSÃO}

O presente artigo apresentou a evolução dos jogos eletrônicos, consoles e PCs, mostrando quais empresas dominavam o mercado nas épocas e alguns dos jogos mais famosos de cada época.

Com isso, observa-se que houve uma evolução imensa no decorrer dos anos e assim mostrando o avanço das indústrias de games.

\section{REFERÊNCIAS}

ASSUNÇÃO, Wolnei. A ascensão dos eSports. Nov. 2018. Disponível em: $<$ https://medium.com/tend\%C3\%AAncias-digitais/a-ascens\%C3\%A3o-dos-esportsa70ce1d9 6ee5>. Acesso em: 23 Jun. 2019.

BARBOZA, Eduardo Fernando Uliana; SILVA, Ana Carolina de Araújo. A evolução tecnológica dos jogos eletrônicos: do videogame para o newsgame. 5o Simpósio Internacional de CiberJornalismo. 27 a 29 Ago. 2014.

BATISTA, Mônica de Lourdes Souza; QUINTÃO, Patrícia Lima; LIMA, Sérgio Muinhos Barroso; CAMPOS, Luciana Conceição Dias; BATISTA, Thiago José de Souza. Um estudo sobre a história dos jogos eletrônicos. 5 Simpósio Internacional de CiberJornalismo. 27 a 29 Ago. 2014.

FOITTE, Vanessa Aparecida; CRUZ, Dulce Márcia. O universo dos jogos eletrônicos: A evolução dos seus personagens, sua narrativa e a fascinação infantil. Aedos. n.12, v.5, Jan./Jul. 2013.

MARQUES, Carla V. M; OLIVEIRA Carlo T; MOTTA, Cláudia L; BARREIRA Christiana V. Games Inteligentes: Investigação Científica por Jogos Computacionais. v.11, n.1, 2015. MOLINA, Lucas Giehl. Jogos digitais como espaço de atuação do historiador: O caso AvantGarde. Revista de Informática Aplicada. ISSN: 1234-5678. v.11, n.1, 2015.

MONTOVANI, Igor. O que é Esports? Saiba tudo sobre esse fenômeno mundial. Mar. 2019. Disponível em: <https://mktesports.com.br/blog/esports/o-que-e-esports/>. Acesso em: 23 Jun. 2019.

PEPE, Felipe. 1975-2014: A Evolução da Indústria dos Computadores \& Video Games. Mar. 2017. Disponível em: <https://medium.com/@felipepepe/1975-2014-aevolu\%C3\%A7\%C3\%A3o-da-ind\%C3\%BAstria-dos-computadores-video-gamesccbee83bb62b>. Acesso em: 14 Jun. 2019.

PINHEIRO, Cristiano Max Pereira. A história da utilização dos games como mídia. Pol. Cult. Rev. Salvador-BA, v.10, n.1, p.237-60, Jan./Jun. 2017.

SILVA, Willians Rodrigues da; MARINS, Luiz Fernando; SANTOS, Éber. Os eventos na era da Informação: um estudo sobre jogos eletrônicos. INTERCOM. XXIV Congresso Brasileiro da Comunicação. Set. 2001.

ZAMBON, Pedro Santoro; CARVALHO, Juliano Maurício de. Origem e evolução das políticas culturais para jogos digitais no Brasil. <https://portalseer.ufba.br/index.php/pculturais/article/view/18226/0>. Acesso em: 13 Abr. 2019. 